\title{
Chopper unit responses to amplitude-modulated tones: does stochastic mode-locking theory allow a more accurate characterisation of observed temporal structure? Jonathan Laudanski*1, Christian J Sumner ${ }^{2}$, Andrew T Wood ${ }^{1}$, Stephen Coombes ${ }^{1}$ and Alan R Palmer ${ }^{2}$
}

Address: ${ }^{1}$ Department of Applied Mathematics, University of Nottingham, UK and ${ }^{2}$ MRC Institute of Hearing Research, Nottingham University Section, University of Nottingham, UK

Email: Jonathan Laudanski* - jonathan.laudanski@maths.nottingham.ac.uk

* Corresponding author

from Sixteenth Annual Computational Neuroscience Meeting: CNS*2007

Toronto, Canada. 7-12 July 2007

Published: 6 July 2007

BMC Neuroscience 2007, 8(Suppl 2):P40 doi:I0.1 I86/I47|-2202-8-S2-P40

(c) 2007 Laudanski et al; licensee BioMed Central Ltd.

Responses to amplitude-modulated pure tones have been used extensively to assess temporal properties of neurons across the auditory system. The synchronisation to the modulation frequency has mostly been measured by an index called vector strength. This index is based on the distribution of spikes along the period of modulation. One obtains a low value when the spikes occur evenly across the period and a high value when they are sharply distributed around a single time. In the ventral cochlear nucleus, chopper units have been found to show band-pass temporal responses at high sound pressure level and low-pass temporal responses at low level [1]. However, the fine structure of the responses remains uncharacterised.

Here, we show that data obtained from chopper neurons in response to amplitude-modulated tone exhibit more complex synchronised discharge patterns, reminiscent of mode-locked states. These responses can be organised around an Arnol'd tongue structure of a periodically forced model accounting for the sub-threshold properties of the T-multipolar cells. Numerical simulations of a stochastic version of this integrate-and-fire model give response patterns similar to the one observed experimentally. Thus, the results tend to show that care should be taken when considering the temporal properties of a neuron only on the basis of its vector strength.

\section{References}

I. Rhode WS, Greenberg S: Encoding of amplitude modulation in the cochlear nucleus of the cat. I Neurophysiol 1994, 7I:I797-1825. 